\title{
Pour une didactique contextuelle, quelques exemples en mathématiques
}

\author{
Alain MERCIER \\ Institut Français de l'Éducation, ENS Lyon
}

\section{Résumé}

Ce texte tente de montrer, sur quelques exemples supposés exemplaires, que la question qui fonde la revue Contextes et Didactique est une question centrale et non pas marginale. On étudiera donc la question du premier enseignement des nombres, un objet dont le caractère universel ne fait aucun doute, en montrant que, pour être efficace, cet enseignement doit être situé explicitement dans un contexte à la fois social et épistémologique, et que la réforme moderniste des années 1970 a conduit à tout le contraire : pourquoi et comment il faut enfin le comprendre parce qu'il est urgent de revenir à un enseignement socialement efficace. Un outil d'analyse didactique sera mis au travail dans une version explicitement anthropologique : la notion de situation fondamentale pour un corps de savoirs.

\section{Mots-clés}

Situation didactique, situation fondamentale, mathématiques, systèmes de nombres, mesures.

\begin{abstract}
This text tries to show on some supposed best examples, that the question underlying the journal Contextes et Didactique is central, not marginal. So we will study the question of the first teaching of numbers, an object whose universality is clear, showing that, to be effective, this teaching should be explicitly situated in a social and epistemological context, and that the modernist reform of the 1970s led to the opposite: why and how it is finally necessary to understand it, since it is imperative to return to a socially efficient education. A didactic analysis tool will be put to work in an explicitly anthropological version: the notion of fundamental situation for a body of knowledge.
\end{abstract}

\section{Keywords}

Didactical Situation, fundamental situation, mathematics, number systems, measures. 


\section{La question de la contextualisation}

Elle est posée par une équipe de recherche située outre-mer, et cela pourrait donner à penser qu'elle se pose " à la marge ». Mais cette question est venue d'un constat, la difficulté spécifique des élèves guadeloupéens à s'investir personnellement dans les questions proposées à l'étude. Cette difficulté ne tient pas seulement à une question de langue vernaculaire, mais bien plutôt, semble-t-il, au fait que les connaissances quotidiennes qu'ils forment dans leur environnement physique et social font obstacle à leur entrée dans les situations d'enseignement que leur proposent les ouvrages pensés en métropole. Cependant en France, la dispersion importante des résultats des élèves aux enquêtes PISA montre que l'on est loin d'y répondre de manière satisfaisante pour tous les élèves ${ }^{1}$. En effet, les élèves les plus faibles sont majoritairement issus des « zones de discrimination positive » et leur écart aux meilleurs élèves, qui eux sont majoritairement issus des établissements d'enseignement privé hors carte scolaire et des établissements des zones d'habitation plus privilégiée, augmente d'année en année tout comme l'écart des revenus et du capital culturel. Ainsi, la question de l'efficacité d'un enseignement républicain qui se proclame " pour tous » est devenue une question centrale. Elle se pose immédiatement à tout professeur de l'enseignement obligatoire public et en particulier à ceux des zones de discrimination dite positive, qui en l'état s'avèrent être bien plutôt des zones socialement discriminatoires.

Ce dont nous avons besoin c'est donc d'une réflexion commune plus que d'une bonne parole sur ce qu'il faut faire ici ou là, dans un temps où les idéologues et les vendeurs de panacée ne manquent pas. Et l'idée que l'enseignement propose aux élèves des situations leur permettant d'agir par eux-mêmes et collectivement pour constituer une communauté de recherche et d'enquête sur des questions ressenties comme importantes semble pertinente pour penser la question. Nous faisons ici référence bien sûr, à Brousseau $(1987,2000)$, mais aussi à Dewey, (1958) et à l'école sociologique de Chicago qui dès les années 1930 a posé des questions proches sur les rapports de l'école à la société et très spécifiquement sur les rapports des questions que les élèves posent aux questions que les pratiques sociales traitent et à celles que la société cherche à résoudre. Ces rapports, nous affirmons que leur analyse et leur compréhension demandent une posture anthropologique relative aux savoirs, qui sont alors considérés comme des pratiques et des techniques vivantes dans un groupe social. Il s'agit en même temps de les faire évoluer selon les besoins sociaux, et de les transmettre sous une forme reconnue, comme compétence personnelle des élèves instruits (Mercier, 2014). C'est la clé de la production de situations didactiques efficaces par des professeurs " professionnels » c'est-à-dire collectivement responsables des contenus de leur intervention enseignante et des effets de cette intervention (Brousseau, 2002 ; Chevallard, 1997, 2007). Nous rejoignons en cela les travaux récents sur l'aide individualisée qui devrait être émancipatrice et s'avère contre-productive :

« Nous formons alors l'hypothèse, à la suite de Dewey, qu'une situation d'enseignement - apprentissage « émancipatrice» est une situation :

1. où l'élève vit une véritable expérience avec le savoir, en ce sens qu'il fait explicitement l'expérience des enjeux de savoir portés par la situation (Dewey, 1975).

2. qui s'inscrit dans un «continuum expérimental », en ce sens que les différentes expériences restent connectées et relèvent d'un même but (Ibid., 1947).

3. où les réussites liées à cette expérience fournissent les règles des prochaines expériences (Ibid., 1975). » (Toullec-Théry et Marlot, 2013)

\footnotetext{
${ }^{1}$ Voir en particulier Bodin et de Franche-Comté (2006) et Bodin (s. d.).
} 
Ces dispositifs en effet sont caractéristiques de l'idéologie pédagogique actuelle et leur étude permet d'en révéler les impasses.

\section{Une question de référence?}

La question qui est posée par la notion de « didactique contextuelle » est donc celle-là même que pose l'idée d'un enseignement fondé sur ce que j'ai appelé «les pratiques anthropologiques des savoirs dans la société ». Il s'agit, ne nous y trompons pas, d'étudier ces pratiques (ce qui se fait dans les écoles, par l'enquête et l'étude) pour en avoir une maîtrise explicite et non pas seulement de les pratiquer de manière compétente (ce qui se fait sur le tas, par frayage). Bien sûr, les étudier doit conduire à en avoir la maîtrise pratique, mais aussi la maitrise théorique afin de pouvoir les faire évoluer. Ces compétences là sont celles d'un citoyen instruit. Il faut considérer alors que définir « ce qu'un citoyen instruit ne peut ignorer et doit avoir été instruit » est un problème d'importance, qui ne peut être traité une fois pour toutes et pour tous les temps, parce que la société change et que de nouveaux problèmes doivent être pris en charge, tandis que certains problèmes anciens deviennent obsolètes.

Le projet a fait en son temps la gloire des penseurs français, depuis le siècle des lumières et l'encyclopédie Diderot puis les textes posthumes de Condorcet jusqu'au siècle de l'école obligatoire et au Dictionnaire de Pédagogie de Ferdinand Buisson. Plus récemment Henri Wallon a tenté de les décrire en psychologue, Dewey a tenté de les définir en philosophe, dans la même période où Freinet le faisait en militant de terrain. Mais tous ont omis un phénomène difficile qui doit nous occuper aujourd'hui : les savoirs du citoyen instruit ont un fonds qui est peut-être stable (nous devrons y regarder de plus près) mais une forme qui est devenue rapidement variable en raison du développement des techniques d'aide à la pensée, de simulation du réel, et de production d'écritures que sont devenus les calculateurs informatiques $^{2}$.

Pour un didacticien des mathématiques, cela peut se dire ainsi : contrairement à ce que porta en avant la réforme moderniste des disciplines qui s'est produite dans les années 1970-1980, le savoir qui fait référence pour l'enseignement et en particulier l'enseignement élémentaire et obligatoire ne peut pas être " le savoir de l'institution savante ", mais il doit bien plutôt se manifester aux élèves et à leurs parents comme " le savoir des pratiques à savoir dans la société ». C'est bien sur ces questions-là, que les élèves ont le droit d'être enseignés et que les professeurs ont le devoir de les enseigner ; c'est sur ces questions là que les auteurs des évaluations PISA tentent d'évaluer les effets de l'enseignement donné et reçu, et qu'ils évaluent ces effets en termes de compétences c'est-à-dire de pouvoir d'agir (Mercier, 2014).

Le travail épistémologique visant à définir les situations sociales nécessitant des savoirs particuliers pour produire les situations didactiques qu'elles peuvent aider à faire vivre, Guy Brousseau 1'a réalisé en cherchant des situations fondamentales pour fonder les éléments de mathématiques à enseigner. Comme le $\mathrm{COREM}^{3}$, que dirigeait Brousseau, n'avait pas accès au Collège mais seulement à l'École pour l'observation Jules Michelet, il a expérimenté ces

\footnotetext{
${ }^{2}$ Ainsi, les Chinois n'utilisent plus de bouliers, même si l'on peut encore en trouver dans les rayons des bazars et des supermarchés. De ce fait tout un répertoire de résultats utiles est devenu obsolète et n'a sans doute plus qu'une existence scolaire, si l'étude des bouliers figure encore dans un plan d'études local. De même, les logiciels de traitement de texte associés aux tablettes peuvent rendre obsolète l'enseignement de l'écriture à la main. En leur temps le stylo à bille puis le feutre ont tué l'écriture à la plume et avec elle, la discipline que l'on appelait l'écriture et qui traitait du graphisme et de « la formation des lettres » minuscules et majuscules.

${ }^{3}$ COREM
} 
situations dans les classes de cette école, en particulier au CM1 et CM2. Pour ce qui est du domaine des systèmes de nombres, le compte rendu de l'expérimentation réalisée de 1980 à 1999 est disponible dans une édition en ligne sur HAL sous le titre Rationnels et décimaux dans la scolarité obligatoire (Brousseau, Brousseau, 1987). En effet Nadine Brousseau les a enseignées longtemps, et elle a observé plusieurs années Denise Greslard qui a eu la charge de la classe de CM2 de l'école ; en 1987, elle en a rédigé un compte rendu précis.

\subsection{Un symptôme possible d'une contextualisation manquante}

Parmi les acquis de la Conférence Nationale sur l'Enseignement des Mathématiques tenue en mars 2012, un résultat sur l'enseignement des entiers, des décimaux, et les difficultés actuelles des élèves, a surpris et interrogé de nombreux participants : aujourd'hui en France, la pratique du système métrique manque aux élèves et aux professeurs qui étudient les nombres (Chambris, 2010). D’abord, ce sont les éléments langagiers associés aux pratiques du mesurage des différents types de grandeurs qui manquent aux professeurs comme aux élèves. Ces pratiques sont donc les formes de vie pour ces jeux de langage. Or, dans un second temps, ces jeux de langage fondaient, dans l'enseignement d'avant la réforme moderniste des années 1970, le discours sur le système décimal; ils fonctionnaient donc comme une théorie de la numération décimale de position (Silvy, Delcroix et Mercier, 2013). La réforme moderniste ayant remplacé cette théorie issue de la pratique par une pratique explicite des systèmes de numération qui servait de forme de vie pour de nouveaux jeux de langage. Les contre réformes ont fait disparaître les conditions de ces pratiques scolaires et les choses ne sont pas revenues à l'état antérieur : ainsi des formes langagières pouvant servir de théorie du système de numération décimale et des décimaux manquent aujourd'hui non seulement aux professeurs, mais aussi bien à leurs formateurs. Le découverte de ce phénomène s'est produite par des chercheurs travaillant dans trois axes différents, liés à la mesure et le mesurage. Brousseau (2002), Le Maréchal et Mercier (2006) travaillent sur la possibilité d'enseigner le volume et son mesurage, au collège; Ligozat (2008) observe comment à l'école les professeurs enseignent les grandeurs en France et en Suisse ; Chevallard et Bosch (2000) montrent le manque, au Collège, des nombres concrets et des grandeurs, qui sont les ostensifs de la modélisation et du contrôle des calculs ; Chambris (2010) engage un travail doctoral sur l'enseignement des grandeurs à l'école et montre la nécessité d'une notion spécifique, elle propose les " unités de numération »; Silvy, Delcroix et Mercier (2013) montrent comment les pratiques de mesurage et d'usage du système métrique servent de discours justificatifs pour les techniques de travail avec les nombres décimaux, et déclarent que le système métrique a fonctionné comme théorie de la notation décimale de position. Cette question nécessite une petite enquête et un peu de théorie, qui en donneront le sens en termes de contextualisation.

\subsection{Le problème : mesurer les grandeurs}

Mesurer est la pratique cognitive première de toute mathématisation. Les cogniticiens ont montré très précisément comment les animaux déjà, les humains ensuite, ont développé, au cours de l'évolution, un système d'évaluation des grandeurs (Dehaene, 2011); mais surtout Carey (2001). Rappelons rapidement les propriétés cette capacité innée à l'évaluation des grandeurs ; elle n'est pas d'emblée numérique, contrairement à ce que laissent croire les auteurs francophones, et elle permet aussi bien de dire qu'une montagne est plus haute qu'une autre (avec un risque d'erreur important si l'une est plus loin que l'autre), qu'un bâton est plus long qu'un autre (et bien sûr le risque d'erreur est plus important si les bâtons ne sont pas disposés parallèlement ou s'ils ne sont pas disponibles au même endroit), qu'un village est 
plus loin qu'un autre (et le risque d'erreur est plus important si les chemins qui y conduisent n'ont pas des configurations semblables), qu'un corps est plus lourd qu'un autre, qu'une durée est plus longue qu'une autre et même, qu'une collection d'objets est plus nombreuse qu'une autre. Pour l'anecdote, je n'ai personnellement compris que compter le nombre des objets d'une collection est une opération de mesure d'une grandeur que lorsque j'ai dû former des professeurs des écoles, et que je me suis posé la question suivante: «Pourquoi les nombres dits « naturels » et les nombres dits « réels » sont tous appelés des nombres? Quel usage ont-ils en commun ?» La différence est donc que les «nombres naturels », ceux qui servent à compter, mesurent une grandeur qui n'est pas nommée et que l'on appellera avec Izard (2006) la numérosité des collections; de ce fait, l'unité de mesure est un individu quelconque de la collection et tous les individus sont équivalents du point de vue du dénombrement, mais l'opération est implicite : 3 pommes et 2 bananes mises ensemble dans une collection forment 5 fruits qui peuvent être indifféremment des pommes ou des bananes, et cette collection de 5 fruits a la même numérosité (mais pas le même goût, ni le même encombrement, ni la même masse, ni la même couleur) qu'une collection de 5 fraises ! Tout cela n'est pas interrogé, sans doute parce que cela semblerait aux praticiens du dénombrement des fruits de mon compotier une interrogation métaphysique : la pratique est donc muette, ce qui n'est pas le cas des autres grandeurs, parce que l'opération de définition d'une unité doit être l'objet d'une convention sociale, qui appartient à chaque société et fait donc partie du contexte des pratiques de mesurage.

Mais les capacités innées ne conduisent pas aux pratiques de mesurage et à l'attribution systématique d'un nombre à chaque grandeur. Le mesurage est un ensemble de techniques sociales spécifiées qui se développent avec l'expérience collective de certaines manières d'organiser le monde (en particulier les systèmes d'échange et le commerce), et il existe donc des communautés humaines qui n'ont pas développé les outils culturels correspondants faute d'intérêt pour les échanges, ou qui sont isolées et n'ont pas d'occasions d'échanges suffisantes. Les pratiques de régulation des échanges par un mesurage conventionnel n'apparaissent semble-t-il que dans les sociétés centralisées où des groupes importants sont réunis dans un système d'échanges complexes et de division du travail, lorsque le troc devient insuffisant. Ainsi, mesurer n'est pas universel et n'apparaît qu'en contexte (Mercier, 1994). Mais dans les sociétés où existent des pratiques commerciales régulées par un pouvoir qui définit les poids les mesures et la monnaie, il faut des écoles pour enseigner la manipulation des mesures de grandeurs que sont les nombres, comptes rendus des mesurages particuliers à chaque espèce de grandeur (Briand et Peltier, 2003; Brousseau, 2002; Brousseau et Brousseau, 1992). Même, il semblerait que certaines espèces de grandeurs et en particulier les grandeurs composées ne sont pas manipulées correctement avant que les techniques de leur mesurage ait été enseignées et qu'une expérience sérieuse de manipulation des mesures obtenues ait été formée : c'est en tous cas une des hypothèses fortes de nos recherches concernant le volume, parce que l'encombrement des objets semble être constitué en obstacle à la conceptualisation de cette notion (Andreucci et Mercier in Le Maréchal et Mercier, 2006); et il est possible que la difficulté soit proche dans le cas des surfaces, qui ne se constituent en grandeur produit que contre le périmètre et par la compréhension de l'intervention du calcul des produits de longueurs dans l'évaluation des aires (Ligozat, 2008).

\subsection{Enquête : La Disme, calcul décimal}

Jusqu'à Stevin (1585) et aujourd'hui encore dans le monde anglo-saxon, on écrit 6 livres $3 / 4$ au lieu de 6,75 livre. Les procédures de calcul sont alors compliquées. Par exemple, pour l'addition de 6 livres $3 / 4$ et de 2 livres $1 / 2$, il faut faire une mise au même dénominateur des 
fractions de livre. Alors, il est plus économique de donner un nom à chaque type de sousunités et par exemple, des onces et des demis, ce qui permet à tous de savoir que un demi c'est deux onces et donc que trois onces c'est un demi et une once, qu'on peut additionner à un demi dès que l'on sait qu'une unité de la grandeur impliquée, la livre, c'est deux demis.

Stevin le premier écrit que les fractions décimales permettent d'éviter tout cela, en particulier en permettant une formation automatique des noms de sous-unités (dixièmes, centièmes, millièmes ; millionièmes, etc.). Ainsi, 6,75 et 2,5 sont 6 unités et 75 centièmes, deux unités et 5 dixièmes, on convertit aisément 5 dixièmes en 50 centièmes, et la somme fait donc 8 unités et 125 centièmes, soit 9 unités et 25 centièmes. Ainsi, la notation décimale des sous unités permet-elle un calcul générique des opérations arithmétiques, selon les mêmes règles utilisées pour les nombres entiers naturels qui sont notés dans le système décimal de position. Stevin l'argumente dans un appendice de moins de trente pages à son traité d'arithmétique intitulé «Die Thiende ». Il sera traduit en français moins de vingt ans plus tard par Girard qui nommera l'appendice : «La Disme » (pages 139-165). L'idée d'un système décimal universel aura en France le succès que l'on sait.

Girard propose d'instaurer un système de sur-unités et de sous-unités décimales pour les mesures d'une espèce donnée de grandeur. On le comprend aisément l'intérêt d'une règle unique cohérente avec le système décimal de position lorsque l'on exécute une simple addition de mesures en toises, pieds, pouces et lignes : 2 toises 3 pieds 5 pouces 2 lignes auxquels j'ajoute 4 pieds 7 pouces 11 lignes cela fait 2 toises 7 pieds 12 pouces 13 lignes; et si une toise fait six pieds, si un pied fait trois pouces et si un pouce fait douze lignes, on obtient ce qu'on appelait «une addition en nombres complexes» pour signifier que le système des unités n'est pas régulier :

\begin{tabular}{rrrrl}
$2 \mathrm{t}$ & $3 \mathrm{P}$ & $.5 \mathrm{p}$ & .21 & \\
+ & $4 \mathrm{P}$ & $.7 \mathrm{p}$ & 111 & \\
\hline $2 \mathrm{t}$ & $7 \mathrm{P}$ & $12 \mathrm{p}$ & 131 & \\
$2 \mathrm{t}$ & $7 \mathrm{P}$ & $13 \mathrm{p}$ & 11 & mais 13 lignes font 1 pouce et 1 ligne. \\
\cline { 1 - 2 } & mais 13 pouces font 4 pieds et 1 pouce.
\end{tabular}

Soit, $2 \mathrm{t} \quad 7 \mathrm{P} \quad 13 \mathrm{p} \quad 11 \quad$ mais 13 pouces font 4 pieds et 1 pouce.

$\mathrm{Ou} \quad 2 \mathrm{t} \quad 11 \mathrm{P} \quad 1 \mathrm{p} \quad 11$ mais 11 pieds font 1 toise et 5 pieds. Après les conversions complètes, on obtient finalement... $3 \mathrm{t} \quad 5 \mathrm{P} \quad 1 \mathrm{p} \quad 11$; nous avons développé le calcul en plusieurs temps car comme les retenues ne sont pas régulières, les réductions à la forme standard sont délicates. Cela pose des problèmes quasi insurmontables au calcul des produits : aires, volumes, densités, vitesses, débits... Pour les volumes, on aurait des toises cubes, des toises carré pieds, des toises pieds carrés, des pieds cubes, avec des rapports de conversion que nous laisserons au lecteur curieux le soin de déterminer, à loisir. Stevin écrit donc en conclusion :

"[...] considerant sa tres grande utilité, ce seroit chose louable, si quelcuns, comme ceux qui en attendent la plus grande commodité, solicitoyent de la faire mettre en effect, à scavoir que joignant les vulgaires partitions qu'il y a maintenant des Mesures, Pois, \& Argent (demeurant chasque capitale mesure, Pois \& Argent, en tous lieux immuable) l'on ordonnast encore légitimement par les Supérieurs, la susdicte dixième partition, à fin que chacun qui vouldroit la pourrait user. Il avancerait aussi la chose, si les valeurs d'argent, principalement de ce qui se forge de nouveau, fussent valuez sur quelques Primes, Secondes, Tierces, \&c. [... $]^{4}$ »

Il nous reste une trace des difficultés de manipulation des nombres complexes avec les calculs mobilisant les heures minutes et secondes : un genre de compétence aujourd'hui presque disparu, mais nécessaire pour calculer des débits ou des vitesses lorsque l'unité n'est pas la seconde, subdivisée en dixièmes, centièmes, millièmes, etc. Avec les systèmes décimaux d'unités et d'écriture des nombres on peut calculer une addition avec les retenues de manière

\footnotetext{
${ }^{4}$ Albert Girard, Les Euvres Mathematiques de Simon Stevin de Bruges (Leiden 1634).
} 
bien plus normalisée et donc automatique, au point que... :

- Il n'est plus utile de noter les unités et sur unités des nombres avec lesquels on calcule... on travaille toujours sur des nombres entiers.

- On arrive donc à calculer sans se préoccuper de l'ordre de grandeur des résultats, que l'on ne détermine qu'après coup...

- Et il devient possible d'oublier que les nombres avec lesquels on calcule dénotent des grandeurs, ce sont des nombres abstraits qui perdent tout sens.

Le sens n'encombre plus le calcul, dont il parasitait l'exécution rapide. Mais on finit par en pâtir, parce que justement c'est le raisonnement sur les ordres de grandeur qui permet de contrôler le calcul, en particulier en physique lorsque les unités se composent. Les professeurs ont alors beau jeu de le reprocher aux élèves et aux étudiants, puisqu'ils ont laissé disparaître les conditions écologiques de la vie de cette notion : eux peuvent bien affirmer qu'ils s'en souviennent, mais l'objet n'a pas même d'existence pour les jeunes générations.

Cependant, l'apprentissage du calcul demande un contrôle des opérations que l'on note et réalise, et voilà que manque une notation qui nous a été bien utile pour le calcul précédent : la notation de ce que Chambris appelle « les unités de numération » et que Stevin avait introduit pour les décimales, considérant que pour les sur-unités il n'est pas utile de noter les ordres de grandeur parce qu'on peut se ramener à un entier (dont la manipulation est supposé connue) en prenant pour référence la plus petite unité. Il ne montre donc explicitement le travail du calcul que pour les décimales dont il note le rang comme une indication d'unité : là où nous écrivons 47,238 il écrit plus explicitement $47_{(0)} 2_{(1)} 3_{(2)} 8_{(3)}$. Nous avons montré (Silvy, Delcroix et Mercier, 2013) que les unités de numération étaient formées sur le mode des sur-unités et des sous-unités, même si les noms ne sont pas formés selon les mêmes règles puisque c'est le grec savant pour les mesures du système décimal issu de la Révolution de $1789^{5}$ et le latin ancien régime pour les noms grammaticaux des ordres de numération ${ }^{6}$, ce qui donne des énoncés explicatifs scolaires étonnants comme «un micromètre, c'est un millionième de mètre $»$.

Ces notations, qui ont disparu avec l'expertise sociale dans la manipulation des décimaux venue de l'introduction du système universel d'unités décimales, sont toujours utiles aux élèves de $\mathrm{CE}$ qui explorent les propriétés de l'écriture décimale de position et notent que $1302 \mathrm{U}=1 \mathrm{M}+3 \mathrm{C}+0 \mathrm{D}+2 \mathrm{U}=13 \mathrm{C}+0 \mathrm{D}+2 \mathrm{U}=130 \mathrm{D}+2 \mathrm{U}=13 \mathrm{C}+02 \mathrm{U}$ (on dit encore « treize cent deux ») pour répondre aux questions du type : "Combien de centaines y a-t-il dans 1302 unités ?» (Il vaudrait mieux dire combien de centaines peut-on former, la réponse est 13). C'est une question que, comme leur professeur, ils ne confondent pas avec: "Quel est le chiffre de l'ordre des centaines dans l'écriture décimale de position 1302 ? " (La réponse est alors 3). Mais on le comprend, ces notations ne vivent plus qu'en CE2, parce que l'introduction de la multiplication permet l'écriture polynomiale des nombres et qu'alors, on écrit tout autre chose :1302 $=1 \times 1000+3 \times 100+0 \times 10+2 \times 1=13 \times 100+0 \times 10+2 \times 1=130 \times 10+2 \times 1$, une écriture qui porte maintenant sur des «nombres abstraits » et ne demande plus les noms de chaque ordre de grandeur ou "unité de compte». Le stade suivant utilise la notation exponentielle des puissances de 10 et complète le processus, en rappelant finalement la proposition polynomiale actuelle, proche de celle de Stevin mais complètement algébrisée :

\footnotetext{
${ }^{5}$ Pour toutes les espèces de grandeurs mesurées en système décimal (mètre, gramme, etc.), on utilise les préfixes déca, hecto, kilo, méga, giga, tera, d'un côté et (au delà de déci, centi, milli, qui appartiennent à la langue courante), micro, nano, pico, de l'autre.

${ }^{6}$ Pour les ordres du système de numération, on utilise les adjectifs dizaines, centaines, milliers, millions, milliards, billions etc. d'un côté et dixièmes, centièmes, millièmes, millionièmes, milliardièmes.
} 
$1302,57=1 \times 10^{3}+3 \times 10^{2}+0 \times 10^{1}+2 \times 10^{0}+5 \times 10^{-1}+7 \times 10^{-2}$. Cependant cet auteur notait encore plus efficacement les ordres seuls, et l'efficacité de sa notation est venue de ce qu'il n'a pas utilisé un appareil descriptif mathématique $\left(10^{\mathrm{n}}, \times\right.$, et +$)$ permettant d'expliciter le " polynôme », ce qui est utile au travail théorique mais inutile à la pratique.

\subsection{Les questions demeurent, leur expression change}

Aujourd'hui, nous affirmons donc que les pratiques du système métrique manquent aux élèves comme aux professeurs. Elles manquent aussi bien pour fonder le discours justificatif des pratiques du système de notation décimale de position que pour fonder les calculs scientifiques de toutes les disciplines, qui portent sur des mesures de grandeurs (Chevallard et Bosch, 2000) Et leur enseignement faut défaut car il rend opaques les pratiques sociales bien réglées des échanges régulés par le système métrique que Stevin, conseiller du Prince d'Orange, appelait de ses vœux. Mais cela ne se voit qu'à ceux qui se mettent en position d'observer très précisément les marchés effectivement passés, et qui ont les lunettes d'une théorie pour voir. En voici un exemple.

En Mauritanie, où la loi ne définit pas ces règles de mesurage, lorsque l'on veut acheter du riz on en demande " une calebasse ». Mais toutes les calebasses n'ont pas la même contenance et il est donc impossible de définir par avance un prix. Les conditions d'un long et difficile marchandage sont réunies et elles vont conduire à de lourdes pertes pour l'une des parties prenantes, le plus faible. En effet, le plus puissant des deux partenaires peut bien refuser les termes d'un accord semblant profitable aux deux parce qu'il peut aller se fournir ailleurs ou vendre à un autre, tandis que le plus faible est dans la nécessité de conclure: les ethnomathématiciens ont observé ce phénomène. Il en va à peu près de même pour les échanges de plus longue portée, lorsque les règles du mesurage ne sont pas identiques sur tout l'espace d'échanges et qu'il faut décider de l'unité qui fera foi. Ainsi, la définition du prix unitaire et son affichage obligatoire régulent les échanges, et cette norme rééquilibre les termes de l'échange, dès lors qu'il n'y a pas entente entre les vendeurs ou entre les acheteurs.

On sait le poids de ces conditions socialement déterminantes (l'entente qui permet de peser sur les échanges) dans les lieux éloignés de la métropole et isolés géographiquement comme les iles : les politiques feignent de l'ignorer, mais les mouvements sociaux de ces dernières années le leur ont amplement rappelé, dans le cas Français. On sait aussi que l'inégalité de l'échange est rendue plus visible par l'usage d'un système universel de mesure et l'affichage des prix unitaires, et le dérégulation européenne actuelle a bien montré ses objectifs de masquage des inégalités le jour où elle a autorisé des emballages de contenance variable. Aujourd'hui pour comparer le prix de deux bouteilles d'huile d'olive, il faut être attentif au fait que leur encombrement peut sembler identique mais que l'une contient $75 \mathrm{cl}$ et l'autre $70 \mathrm{cl}^{7}$, et qu'il faut donc se référer au «prix au kilogramme » affiché en petits caractères. De la même manière, la vente par lots de deux ou trois bouteilles affichant «la troisième est gratuite » cache le fait que la réduction est de $33 \%$ et triple la vente, ce qui double le chiffre d'affaires et, très probablement, maintient le bénéfice du «distributeur » et sa position dominante sur le marché.

La fonction sociale des mathématiques est la source de problèmes très concrets qui étaient connus des anciens auteurs de programmes, comme le montre cet exemple pris dans le manuel de Pugibet, Adam et Gason (1948: 271) : Un ouvrier agricole achète 750 l de cidre

\footnotetext{
${ }^{7}$ Bouteille trompeuse déjà utilisé au XVIII ${ }^{\circ}$ siècle : on en trouve un exemplaire dans le musée de Marie Galante.
} 
" pur jus » au prix de 220 fl'hectolitre. Combien devra-t-il ajouter d'eau pour que le litre de mélange lui revienne à $1 f 50$ ? Ces problèmes sont bien sûr obsolètes, les pratiques d'échange commercial ont profondément changé. Mais sur les forums d'aide aux devoirs, on trouve aujourd'hui une question qui mobilise des connaissances semblables bien qu'elles ne soient plus enseignées : Je n'arrive pas à résoudre la question suivante, un laboratoire dispose de 2 solutions de chlorure de sodium, de concentration respective 20 g.L $L^{-1}$ et 50 g.L. $L^{-1}$. Quelle quantité de chaque solution doit-on mélanger pour obtenir $10 \mathrm{~L}$ d'une solution de concentration 32 g. $L^{-1}$ ? Merci pour votre aide. (Tina, élève de 3e, mai 2012). Ces deux problèmes bien concrets dans les situations décrites demandent pour être traités la manipulation explicite et réglée des noms d'unités, qui donnent le contrôle des calculs conduits et garantissent la pertinence de la réponse donnée par une calculatrice. De plus, ces deux problèmes relèvent des " problèmes de mélanges » et de densité ou de concentration, or la densité comme la concentration est mesurée par une unité composée, et les mélanges relèvent du calcul des moyennes pondérées ou barycentres, qui n'est plus enseigné en mathématiques $^{8}$. Ainsi, l'École ne joue plus son rôle social parce que les contenus d'enseignement ne sont plus pertinents pour le traitement de questions vivantes dans la société, c'est-à-dire parce que l'enseignement devient massivement décontextualisé, sur tout le territoire national. Ainsi, les contre réformes d'après la réforme moderniste des années 1970 n'ont pas réussi à produire de nouveau un enseignement contextuel, pas même en métropole et encore moins outremer; il aurait fallu pour ce faire un travail long, patient, et permanent d'enquête sur les problèmes du quotidien et les pratiques correspondantes, qui se renouvellent aujourd'hui de manière accélérée. Faute de cela, les tenants de «l'école républicaine » (l'enseignement primaire supérieur) qui a fait d'eux des membres de l'élite sociale pensent que les questions qu'ils ont traitées dans leur enfance sont la panacée, ce qui les rend réactionnaires et contre productifs.

\subsection{Exemples de l'évolution des pratiques sociales mobilisant la mesure des grandeurs}

Les surfaces habitables des appartements sont déterminées aujourd'hui à l'aide d'un instrument à visée laser infrarouge, par trois prises d'information depuis le centre d'une pièce, l'une qui prend une « largeur » l'autre une « longueur » approximativement perpendiculaire et la troisième qui vérifie que la hauteur est supérieure au minimum requis. Le lecteur intéressé estimera la précision de l'opération pour des pièces réelles qui ne seraient pas exactement rectangulaires, et dans quels cas la pratique donne bien une erreur relative inférieure à $5 \%$ par excès comme c'est prescrit par la loi Boutin. On notera, pour une analyse rapide, que si le métreur se tient au centre d'une pièce, nul besoin qu'elle soit rectangulaire, les parallélogrammes relèvent de la même formule (pourvu que les deux mesures soient prises selon la direction des côtés parallèles et perpendiculairement), les trapèzes aussi peuvent être mesurés ainsi (par le produit des deux dimensions que sont leur hauteur, prise entre les deux côtés parallèles, et leur longueur moyenne, qui se mesure justement au milieu de leur hauteur). Les triangles sont des trapèzes particuliers. Restent les pièces qui ne sont pas définies par trois ou quatre murs droits dont deux au moins seraient parallèles, et dont l'aire doit être décomposée en somme de celle des deux triangles définis par une de leurs diagonales.

\footnotetext{
${ }^{8}$ La seule préconisation est «Des travaux réalisés à l'aide d'un logiciel permettent de faire observer des exemples d'effets de structure lors du calcul de moyennes ». On la retrouve en seconde et première. Le barycentre n'est donc plus même au programme de première scientifique.
} 
Un tout autre type d'exemple nous est donné par les modes que les fabricants de montres ont tenté de créer: les premières montres électroniques ont en effet mis en avant l'affichage digital de l'heure, aujourd'hui disparu des poignets après que les Swatch aient choisi avec succès un affichage analogique. Que s'est-il passé ? Traditionnellement, l'heure se lit sur une graduation, l'extrémité des aiguilles décrivant un parcours circulaire gradué en 12 heures pour l'une (graduation intérieure, petite aiguille) et cinq fois plus soit 60 minutes ou secondes pour les deux autres (graduation extérieure, grandes aiguilles plus fines). Ainsi, la lecture des instruments à cadran est toujours lecture d'un point désigné par une aiguille sur une graduation, que la ligne soit droite ou cercle ne change rien à la technique, mais le fait que le cadran soit circulaire permet des énoncés fort utiles : « Il est trois heures moins le quart» est une annonce sur le fait qu'on est peu de temps avant «trois heures » et c'est une évaluation d'attente peu coûteuse à produire, bien suffisante et utile lorsque le temps ne demande pas de mesure plus précise. Tandis que donner un rendez-vous à « trois heures moins le quart» et pour cela régler adéquatement l'alerte de sa montre à affichage digital sur 14 heures 45 minutes pose un problème dont le traitement produit de nombreuses erreurs. Cependant aujourd'hui il est probable que beaucoup utilisent leur smartphone avec de nouveaux modes pour gérer leurs rendez-vous : les montres ont-elles encore un usage autre que ce qu'à Marseille on appelle « la montre » c'est-à-dire le paraître ?

Les usages du temps ont donc imposé divers affichages de l'heure, lire l'heure demeure sans doute un problème à enseigner aux enfants dans les écoles, sans que l'on sache précisément ce que contient cette compétence. Mais pour toutes les civilisations qui vivent sur la coordination précise des activités et où un moyen collectif d'alerte (comme les clochers ou les minarets qui appellent à la prière, ou les sirènes qui alertent d'un incendie) ne suffit pas aux besoins quotidiens, soit lorsque le temps est compté pour d'autres usages que la religion ou l'organisation militaire du travail.

\section{Comment produire un enseignement contextuel ?}

Comme les exemples précédents le montrent, inventer un enseignement contextuel ce n'est pas seulement une question de référence à des pratiques sociales. Car les questions sur lesquelles il peut être pertinent d'enquêter et les questions mathématiques qu'il peut être utile d'étudier ne sont pas tout naturellement des questions instructives, permettant d'apprendre des mathématiques ou permettant d'en pratiquer de manière intéressante. Pour aller plus loin dans ce sens il nous faut d'une part, faire appel à ce que nous savons des conditions de vie de l'étude collective et de ses formes d'organisation (les conditions didactiques d'un apprentissage) et d'autre part, faire appel à ce que nous savons de l'écologie scolaire des savoirs (les conditions sociales de l'introduction ou de la disparition d'un objet enseignement $\left.{ }^{9}\right)$.

Nous tenons la position qui, selon Fabre (2015) ou Go (2009), est celle de Dewey, pour qui une école démocratique est fondée sur les intérêts personnels et sociaux des enfants qu'elle reçoit et sur ceux de leurs familles, parce que la clé de la réussite à l'école est dans cet intérêt, qui seul garantit un mouvement personnel fort en direction de l'enquête sur le monde et ainsi, la formation des connaissances socialement visées et utiles, par le plus grand nombre (Mercier, 1994 ; Mercier, 2000).

\footnotetext{
${ }^{9}$ Ainsi, les «problèmes du second degré » n'ont plus de motif à être enseignés, mais personne n'ose les faire disparaître, tout comme les «techniques du calcul posé » qui vont cependant très probablement résister, et survivre dans les futurs nouveaux programmes des cycles 1 à 4 .
} 


\subsection{Enquête sur le monde}

C'est la notion d'enquête qui nous permet de déclarer fermement que toute position didactique doit être contextuelle; Dewey la situe au fondement de la compréhension des phénomènes matériels et sociaux dans une société démocratique capable d'évolution raisonnable, et donc au centre de tout enseignement. Nous savons que ce type de questionnement était l'enjeu de l'enseignement élémentaire en France il y a cinquante ans, et que cela s'est perdu. Nous savons aussi que les objets d'enquête étaient prédéfinis et qu'ils faisaient sens plus aisément en métropole qu'outremer. Mais nous la reprenons à notre compte pour discuter de la notion de situation et de ses déclinaisons (action, formulation, validation), qui semble désigner une enquête scolairement organisée visant la formation de savoirs et leur acquisition, ce qui signifie que l'enquête est spécifiée lorsqu'elle est orientée par sa fonction de moyen d'enseignement, mais ne dit pas immédiatement que l'enquête doit, dans l'enseignement obligatoire, être orientée par les pratiques sociales dont l'intelligibilité doit être assurée.

Or, on observe que la «ligne des nombres » vient dès la maternelle à être proposée aux élèves. Mais peut-on dire et attester du fait qu'elle permet d'anticiper, par exemple, la manipulation d'une règle graduée pour mesurer une longueur ou un écart entre deux longueurs, l'usage d'un calendrier pour déterminer une date ou la durée d'une période scolaire, d'une montre pour anticiper une durée ? Rien n'est moins sûr, d'abord parce que les nombres y sont écrits dans les cases et non pas en face des points de graduation (la ligne des nombres du type «jeu de l'oie » propose donc un modèle "discret» qui n'anticipe pas l'évaluation d'une grandeur continue) et ensuite, parce qu'elle est affichée sur un mur et ne peut donc être manipulée pour montrer la mesure d'une différence ou d'un ajout. On ne peut donc que compter les cases et c'est un problème parce que, de ce fait, l'addition et la soustraction n'apparaissent pas comme modèles des manipulations de comparaison de deux grandeurs de même espèce. La ligne des nombres n'anticipe pas les opérations de mesurage et ne permet donc pas de former une connaissance utile des nombres.

Un instrument de mesure à affichage analogique transforme toute grandeur en longueur (longueur d'un arc sur une graduation circulaire ou longueur d'un segment sur une ligne droite) et la graduation décimale de cette ligne (arc ou droite) donne par simple lecture un nombre décimal qui est la mesure cherchée. Lorsque l'on observe des classes d'aujourd'hui en anthropologue des pratiques scolaires, ce sont la pratique des graduations pour définir les nombres et la pratique des lignes pour représenter les grandeurs qui manquent. Une enquête sur les graduations des règles, rapporteurs, et autres cadrans aurait permis une meilleure connaissance et l'accès à des problèmes additifs (se résolvant par addition ou soustraction c'est-à-dire par une technique de manipulation d'une règle sur une graduation) bien plus intéressants.

Cependant, l'usage des instruments de mesurage n'est plus au centre des programmes comme ce fut le cas naguère. Voici en exemple la table des matières mathématiques et la table générale des questions étudiées de l'Arithmétique en 1948, selon deux Inspecteurs primaires et un inspecteur général (voir tableau 1). 


\begin{tabular}{|c|c|}
\hline $\begin{array}{l}\text { Numération parlée et écrite .... } \mathrm{p} 1 \text { à } 2 \text { et } \mathrm{p} 3 \\
\text { Nombres et grandeurs ..................1 page } \\
\text { Premières unités de mesure ........... } 3 \text { pages }\end{array}$ & \multirow{2}{*}{$\begin{array}{l}\text { Problèmes pratiques, peintures carrelages papiers peints } \\
\text { boiseries doublures .4 pages } \\
\text { La division.........p } 109 \text { et } 110,113 \text { et } 114 \\
\text { Applications ............................... pages }\end{array}$} \\
\hline $\begin{array}{l}\text { La ligne droite, segments de droite } \mathrm{p} 10 \text { à } 11 \\
\text { Usage de la règle .......................1 page }\end{array}$ & \\
\hline 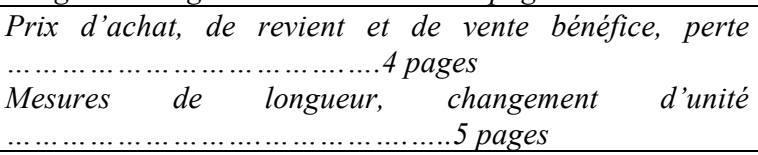 & 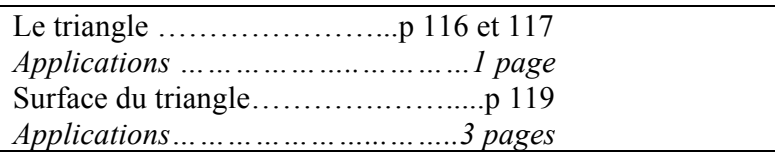 \\
\hline $\begin{array}{l}\text { Nombres } \quad \text { décimaux, } \quad \text { fractions } \\
\ldots \ldots \ldots \ldots \ldots \ldots \ldots \ldots \ldots \ldots \ldots \ldots \ldots \text { p } 22 \text { et } 23 \\
\text { Applications } \ldots \ldots \ldots \ldots \ldots \ldots \ldots \ldots \ldots \ldots \ldots \ldots \ldots \ldots \ldots \ldots \ldots \ldots \ldots \ldots \ldots \\
\end{array}$ & Problèmes pratiques, le mobilier ...2 pages \\
\hline Valeur des marchandises...............3 pages & $\begin{array}{l}\text { Propriétés des quotients........p. } 125 \text { et } 126 \\
\text { Applications ........................1 page Divisibilité par } \\
2,5,3,9 \ldots \ldots \ldots \ldots \ldots .128\end{array}$ \\
\hline Les angles, angle droit ............p 29 à 33 & \\
\hline 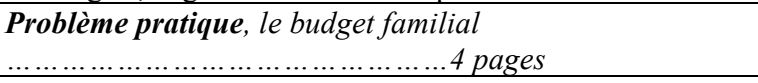 & \multirow{2}{*}{$\begin{array}{l}\text { L'ouvrage conserve le même rythme tout au long de ses } 350 \\
\text { pages d'explications, trois pages d'exercices... } \\
\ldots \text { et environ toutes les } 20 \text { pages, un thème pratique dont } \\
\text { nous donnons le titre. }\end{array}$} \\
\hline $\begin{array}{l}\text { Rendre un nombre } 10,100,1000 \text { fois plus grand ou plus } \\
\text { petit ..............p } 37 \text { et } 38 \\
\text { Applications.......................2 pages }\end{array}$ & \\
\hline $\begin{array}{l}\text { L'addition, sens de l'opération ......p } 40 \text { à } 42 \\
\text { Les poids les balances................5 pages }\end{array}$ & \\
\hline $\begin{array}{l}\text { Tracé des perpendiculaires, médiatrice d'un segment } \\
\ldots \ldots \ldots \ldots \ldots \ldots \ldots \ldots \ldots \ldots \text { p ..4 } 48 \text { à } 51\end{array}$ & $\begin{array}{l}\text { Problème pratique, le remembrement des propriétés, les } \\
\text { échanges.................4 pages }\end{array}$ \\
\hline 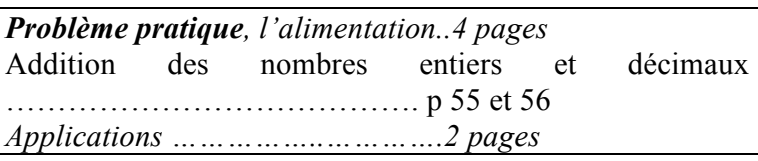 & 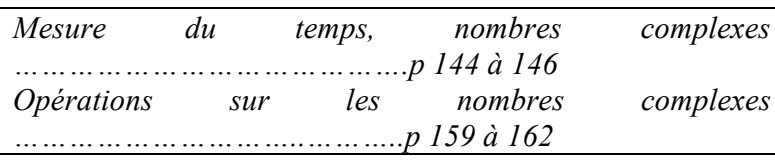 \\
\hline 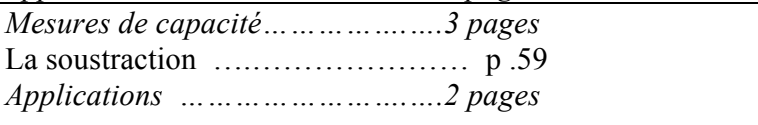 & 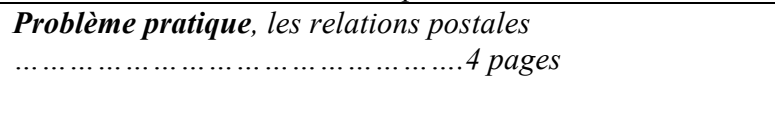 \\
\hline $\begin{array}{l}\text { Droites parallèles } \ldots \ldots \ldots \ldots \ldots \ldots \ldots \ldots \ldots \ldots \ldots \\
\text { Applications...........................2 pages } 65 \text { à } 67\end{array}$ & $\begin{array}{l}\text { Vitesse débit chemin parcouru, division et multiplication } \\
\text { par un nombre complexe ......................................p } \\
163 \text { à } 165 \\
\text { Calcul du temps division des nombres } \\
\text { complexes..........................p } 166 \text { à } 168\end{array}$ \\
\hline $\begin{array}{l}\text { Soustraction des nombres décimaux ...p } 74 \\
\text { Applications ............................ pages }\end{array}$ & \multirow{2}{*}{$\begin{array}{l}\text { Problème pratique, fuseaux horaires longitude et latitude } \\
\text { mesures marines. } 3 \text { pages } \\
\text { Le cube le mètre cube unités de volume } \\
\text {.............................................pp } 203 \grave{a} 20\end{array}$} \\
\hline $\begin{array}{l}\text { La multiplication................ p } 77 \text { et } 78 \\
\text { Applications ........................2 pages }\end{array}$ & \\
\hline $\begin{array}{l}\text { Le rectangle le carré, le périmètre..4 pages } \\
\text { Les mesures de surface................... } 3 \text { pages }\end{array}$ & Problème pratique, les transports ... 5 pages \\
\hline 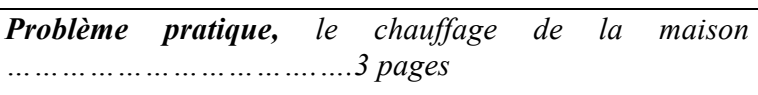 & 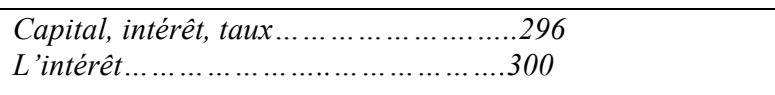 \\
\hline 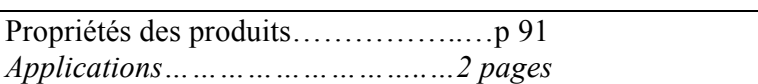 & Poids spécifiques............................303.. \\
\hline $\begin{array}{l}\text { Multiplication des nombres décimaux et des fractions } \\
\text { décimales ....................p } 94 \\
\text { Applications........................2 pages }\end{array}$ & 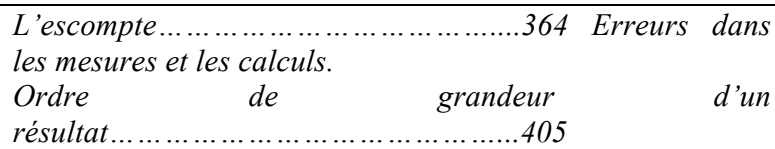 \\
\hline $\begin{array}{l}\text { Mesures de surface mesures agraires } \\
\ldots \ldots \ldots \ldots \ldots \ldots \ldots \ldots \ldots \ldots \ldots \ldots \ldots \ldots \ldots \ldots . . \ldots \ldots \text { pages } \\
\text { Surface du rectangle, du carré. } \mathrm{P} 100 \text { et } 101\end{array}$ & Problèmes à données surabondantes......409 \\
\hline
\end{tabular}

Tableau 1 : Analyse de la table des matières (Pugibet, Adam et Gason, 1948) 
On remarque que même les problèmes « moins pratiques » apparaitraient aujourd'hui comme absolument «concrets » bien qu'ils ne réfèrent plus à des actes relevant de nos jours d'un calcul à la main. Mais il serait sans doute intéressant de voir comment une enquête organisée sous la direction du professeur permettrait d'obtenir des problèmes de calcul ou d'estimation effectifs c'est-à-dire rencontrés au quotidien par les pratiquants des outils numériques de réponse automatisée sur l'escompte, le temps, les questions de vitesse ou de débit, les relations postales ou le calcul de l'impôt, le calcul des carrelages ou des prix de revient, les mesures de la terre, etc. On sait en tous cas (Dutheil, 1996) que l'une des difficultés des « enfants d'ouvriers » à apprendre des mathématiques tient au fait qu'elles ne «sont pas concrètes ».

\subsection{Quelques problèmes sur lesquels enquêter}

Les problèmes sur lesquels il serait possible d'enquêter sont pourtant légion, si l'on en croit les revues proposant de telles enquêtes aux élèves éclairés parce que leurs parents sont abonnés, comme Tangente pour le Lycée, en France, ou la revue canadienne Accromaths. Mais est-ce aussi le cas de l'École Élémentaire et du Collège ? A ces niveaux, l'enquête doit être conduite dans un esprit anthropologique plus que mathématique, car elle doit en venir à des questions pratiques devenues invisibles aux mathématiciens. Nous ne traiterons ici que de deux cas, en montrant comment des questions simples peuvent fonder une enquête et des études sur une longue période, si ce type de travail correspond à un projet soigneusement construit pour donner lieu, par exemple, à une exposition de fin d'année en direction des autres élèves de l'établissement et des familles environnantes.

\subsubsection{Pourquoi les éléphants sont plus petits que les baleines?}

Une telle affirmation se situe dans un espace de questionnement large, que l'on peut définir sans le construire en ouvrant d'autres questions du même type comme celles-ci : «A quoi ressemblerait un géant de 4 mètres? » (Une réponse peut se trouver dans un numéro de Accromaths), ou inversement, "Pourquoi le plus petit des mammifères terrestres est-il bien plus gros qu'un insecte?, Et pourquoi est-ce encore plus vrai dans le cas des mammifères marins ? » Mais encore : " Pourquoi le plus gros oiseau qui puisse voler pèse-t-il à peine plus de dix kilogrammes ? » (On peut facilement constater qu'un albatros, 3,5 $\mathrm{m}$ d'envergure pour 12 kilos, plane presque tout le temps sans battre des ailes ; est-ce qu'il ne pourrait pas décoller sans les vagues et le vent des mers australes ?) Mais alors, "Quels sont les plus gros animaux ayant jamais volé ?»Ce seraient peut-être les Quetzalcoaltus, 4 mètres de long pour 12 d'envergure. Leur poids a été évalué à 250 Kilos et alors, il leur aurait été impossible de décoller en battant des ailes selon les simulations informatiques; mais s'ils avaient pesé 70 kilos grâce à des os creux, ils auraient pu planer en décollant face au vent sur une pente descendante, ailes ouvertes, comme les deltaplanes, ne se posant que très rarement et sur des sommets. On aurait pu trouver encore : «Comment les gros bateaux en fer flottent-ils ? » et la question associée mais plus difficile à imaginer : "Pourquoi les petits bateaux ne sont pas en fer?» Sur les questions relatives au vivant, seule une enquête sur internet ou dans une encyclopédie permet d'obtenir des renseignements, mais, sur la flottabilité, il est possible d'ouvrir une expérimentation qui conduise à un questionnement mathématique. Cela suppose en revanche l'idée que les mathématiques à étudier doivent venir à point pour nourrir une enquête ouverte par ailleurs, et non pas permettre de résoudre des problèmes ad hoc. Cela suppose donc des thèmes et des sujets d'enquête disponibles aux professeurs. Imaginons rapidement que l'on s'engage dans une tentative de trouver réponse à l'une des questions 
posées, de manière à comprendre de quelles mathématiques ces questions relèvent, selon le type de réponse que l'on cherchera à asseoir. Prenons la question qui nous a servi de titre, relative à la comparaison de l'éléphant et de la baleine.

1) Le professeur peut diriger et organiser la classe afin qu'elle conduise une enquête dans une encyclopédie ou sur internet: l'assertion est-elle vraie, quelle est sa valeur de généralité ? L'assertion est vraie et si un éléphant mâle adulte pèse généralement de 4 à 7 tonnes, mais certaines baleines peuvent peser bien plus et jusqu'à 200 tonnes. Alors, pourrait-on imaginer des animaux terrestres de ce poids ? Quel est le problème qui se poserait à eux ? Pour le comprendre on peut comparer l'éléphant au chat, bien plus petit et léger puisqu'il pèse plutôt 4 à 6 kilos, soit 1000 fois moins qu'un éléphant. On peut le vérifier en pesant quelques chats disponibles, à l'école ou au domicile des enfants ce qui mobilise les parents : on verra que le plus efficace est de se peser avec et sans le chat, puis de calculer la différence, car les balances ne sont pas bien sensibles et donc fiables, pour des poids faibles; on pourra calculer un poids moyen et un intervalle de confiance pour dire quelque chose comme "un chat domestique normal pèse $\mathrm{x}$ kilogrammes », et trouver dans une encyclopédie une réponse de ce type pour les éléphants.
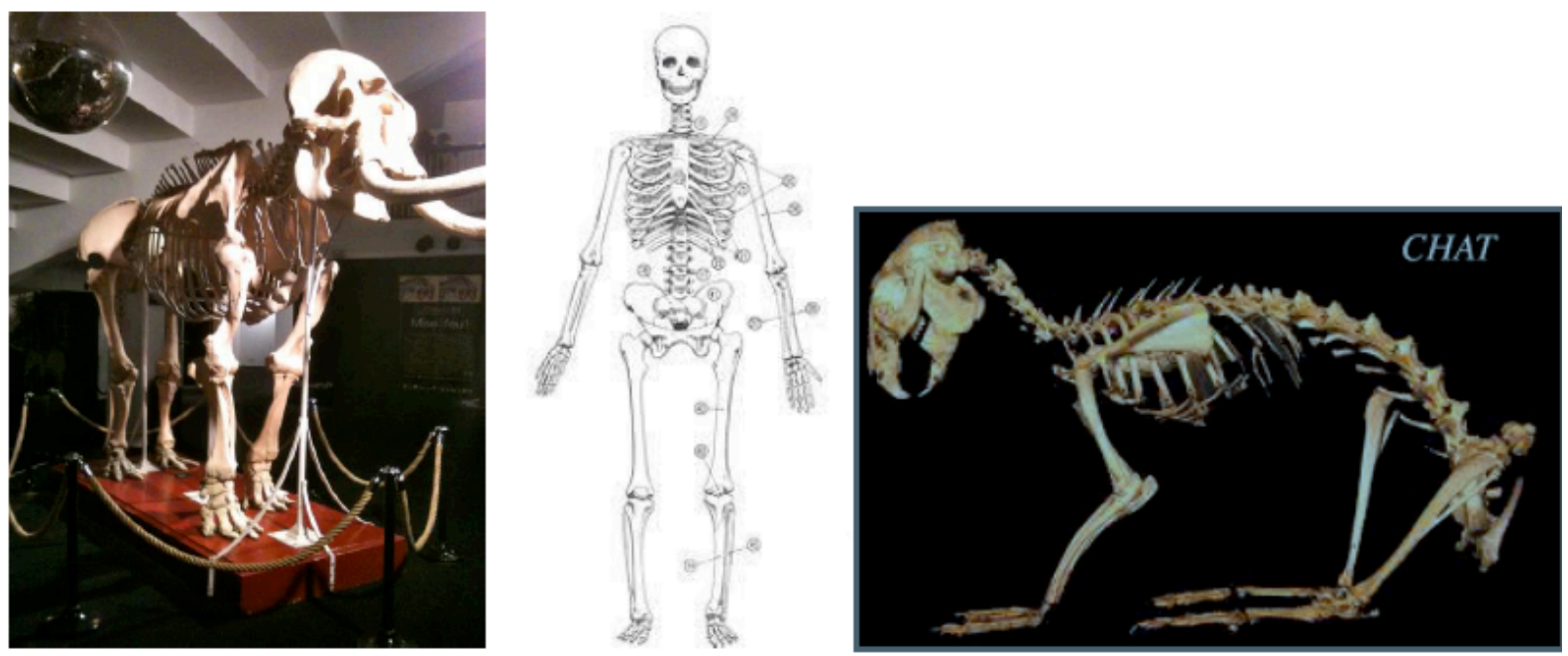

Figure 1 : les squelettes

2) Le professeur peut alors proposer d'étudier une question scientifique : Peut-on évaluer la solidité du squelette d'un vertébré, afin de la mettre en rapport avec la masse de l'animal? Sans doute, mais il faut peut être s'étonner d'abord du rapport des poids d'un éléphant et d'un chat, s'il s'avère vraiment qu'il est de 1 à 1000. Car le rapport des tailles semble bien inférieur ; on penserait qu'un chat ne mesure guère plus de $30 \mathrm{~cm}$ au garrot et $45 \mathrm{~cm}$ de long pour moins de $10 \mathrm{~cm}$ de large, ce qui lui permettrait de rentrer dans une boîte de cette taille, tandis qu'un éléphant mesure plus de 3,5 mètres de haut pour au moins 3,5 mètres de long et moins de deux mètres de large, ce qui lui permettrait de rentrer lui aussi dans une boîte de cette dimension. Un éléphant a donc des dimensions environ dix fois supérieures à celles d'un chat. Cependant, quel est le rapport des volumes des deux boîtes ? 0,3m $\times 0,45 \mathrm{~m} \times 0,12 \mathrm{~m}$ soit environ $0,016 \mathrm{~m}^{3}$ et $3,5 \times 3,5 \times 1,5$ soit environ $18 \mathrm{~m}^{3}$, le rapport des volumes est de l'ordre de $0,016 / 18$, ce qui est de l'ordre du rapport de 0.9 à 1000 , et qui confirme le rapport des poids. Bref, il faut pour que l'animal marche en gardant toujours au sol deux pattes diagonales, que chaque patte de l'éléphant porte de 2000 à 3500 kilos tandis que chaque patte du chat porte environ de 2 à 3 kilos. A l'arrêt, chaque jambe humaine porte la moitié du poids soit moins de 50 kilos, mais le double lors de la marche. Peut-on voir si cela est vérifié en observant les os et les muscles? Sans doute, comme le montrent les photos ci-dessus. 

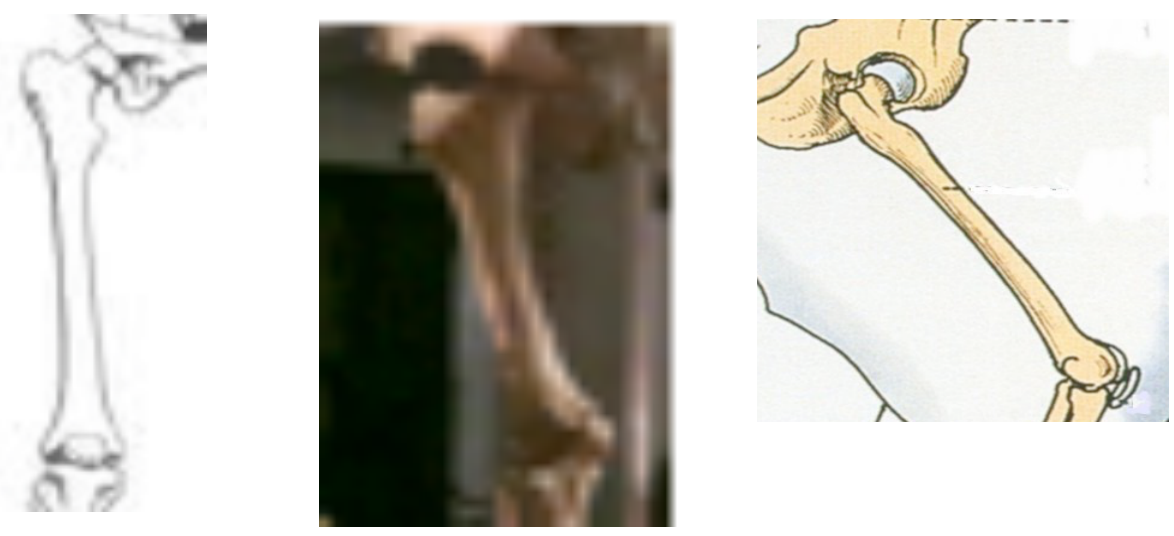

Figure 2 : les fémurs

Si en effet nous voulons comparer les trois squelettes en agrandissant les plus petits à la taille de celui de l'éléphant, alors nous obtenons ceci : les os de l'éléphant sont bien plus massifs que ceux de l'homme, qui semblent eux-mêmes plus massifs que ceux du chat, ce que l'on observe en comparant par exemple le fémur. Voici un fémur humain, un fémur d'éléphant et un fémur de chat (figure 2), dont on peut tenter de comparer le rapport longueur/section en mesurant sur les photos à l'échelle le diamètre (d'où la section $s=\pi . d^{2} / 4$ ) et la longueur.

\begin{tabular}{|l|l|l|}
\hline Homme : & Eléphant : & Chat : \\
\hline $\mathrm{L}=90, \mathrm{~d}=8$ & $\mathrm{~L}=90, \mathrm{~d}=12$, & $\mathrm{L}=90, \mathrm{~d}=6$ \\
$\mathrm{~s}=50$, & $\mathrm{s}=113$, & $\mathrm{s}=28$, \\
$\mathrm{s} / \mathrm{l}=0,55$ & $\mathrm{~s} / \mathrm{l}=1,25$ & $\mathrm{~s} / \mathrm{l}=0,3$ \\
\hline
\end{tabular}

Tableau 2

Ainsi (voir tableau 2), le fémur de l'éléphant est presque deux fois et demi plus massif que celui de l'homme qui est lui-même deux fois plus massif que celui du chat, tandis que les rapports des masses sont d'un tout autre ordre $(1,5 ; 50: 1500)$. On comprendra que l'éléphant ne puisse pas comme l'homme entraîné se recevoir d'un saut de deux fois sa hauteur en amortissant sa réception grâce aux muscles de ses cuisses, ni même courir ou sauter (il doit toujours avoir au moins une patte posée pour se soutenir), tandis que le chat peut survivre à une chute libre importante et saute cinq fois sa hauteur. Un animal du même type (un vertébré mammifère) plus pesant encore que l'éléphant ne pourrait plus que marcher avec précautions sur un sol spongieux qui amortirait ses pas. Les sections des mêmes os, si l'on enlève la mise à l'échelle que le travail des images permet et si on mesure sur les images agrandies de la première figure sont dans un rapport qui est approximativement de $(1,22,160)$ ce qui n'est pas encore le rapport des masses de ces trois mammifères, mais est fort intéressant lorsque l'on s'aperçoit que le fémur de l'homme est à peu près de la même longueur que celui de l'éléphant: alors qu'il porte au repos un poids 40 fois moindre, il est seulement sept fois moins épais et il est donc environ sept fois plus solide (c'est d'ailleurs on le sait le col du fémur, qui est le point fragile). D'autres questions arrivent alors : Il semblerait que les gros dinosaures vivaient dans des marécages ou étaient bipèdes, peut-on faire le lien entre ces propriétés et leur masse ? Pourquoi n'y a-t-il pas de mammifères aussi petits que les insectes et d'ailleurs, pourquoi n'y a-t-il pas de très gros insectes ? Etc.

3) Enquête: dans l'eau, combien pèse une baleine ? La réponse peut étonner : elle ne pèse rien, bien que sa masse soit grande. De ce fait les seuls efforts que supporte son squelette tiennent à son activité natatoire c'est-à-dire aux efforts qu'elle exerce sur l'eau. Ceux là sont 
plus difficiles à évaluer simplement, et nous remettrons l'enquête aux bons soins des lecteurs intéressés, mais il semble évident qu'ils sont moins importants que ceux qui lui seraient nécessaires pour marcher.

On notera que le premier à avoir travaillé sur la variation de la forme des os, plus massive chez les animaux les plus imposants, est Galilée, qui a identifié ces effets que nous appelons aujourd'hui «effets d'échelle». Ils tiennent à ce que les fonctions de degré 1 , 2, et 3 ne varient pas de la même manière, autrement dit que la suite 1,2,3,4,5 correspond à la suite des carrés $1,2,9,16,25$ et à la suite des cubes $1,8,27,64,625$ ce qui fait qu'une variation au degré 1 (la longueur) peut correspondre à une variation bien plus importante au degré 2 (l'aire) et devient sans commune mesure au degré 3 (le volume ou la masse). Nous allons en proposer un second cas, afin de montrer que des mathématiques élémentaires peuvent permettre de traiter de questions essentielles pour la compréhension de phénomènes du vivant comme de phénomènes techniques, et nous conclurons en donnant une liste de phénomènes qu'il est aisément possible d'investiguer lorsque le phénomène d'échelle est reconnu.

\subsubsection{Comment les gros bateaux en fer flottent-ils?}
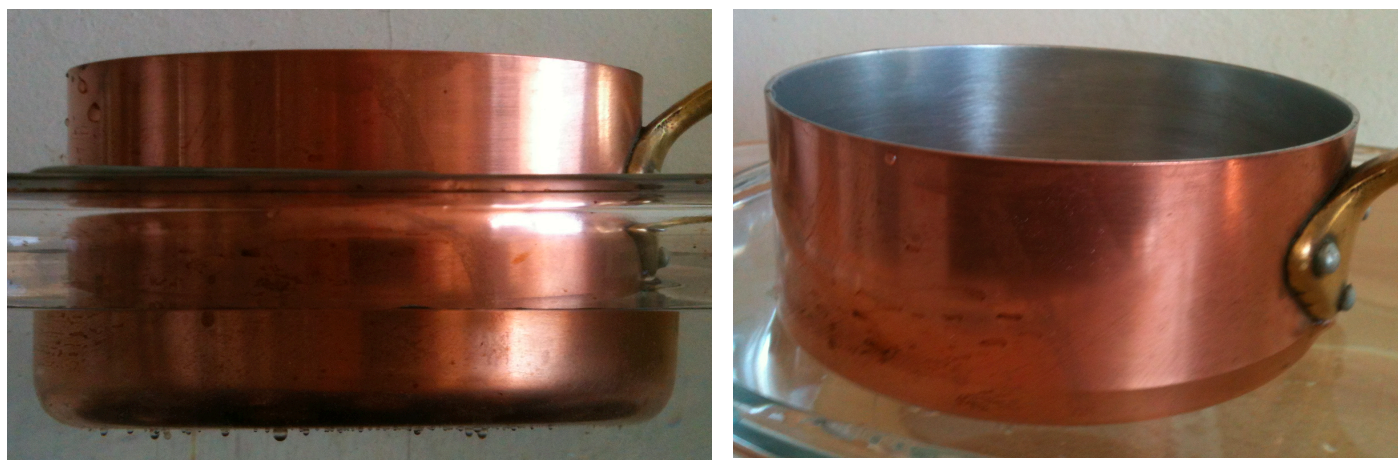

Figure 3 : casseroles dans de l'eau

Ces deux casseroles (figure 3) ont été mises dans un bac d'eau, manifestement l'une « flotte moins bien $» .$. pourtant elles sont faites du même matériau, de même épaisseur (du cuivre). D'où vient la différence?

Si nous savons que l'enfoncement d'un corps est proportionnel à sa masse, nous pouvons proposer une formule de calcul de la masse : les casseroles sont composées d'un fond en forme de disque de diamètre $\mathrm{d}$ d'aire $\mathrm{s}=\pi \mathrm{d}^{2} / 4$ et de volume $\mathrm{v}_{\mathrm{f}}=\mathrm{se}$ (si nous appelons e l'épaisseur du cuivre), et d'un tour de même épaisseur et que l'on peut considérer comme un rectangle de hauteur $\mathrm{h}$ et de longueur $\pi \mathrm{d}$, donc de volume $\mathrm{v}_{\mathrm{c}}=\pi \mathrm{dhe}$, la casserole complète étant donc constituée d'un volume $\mathrm{V}$ de cuivre égal à $\mathrm{v}_{\mathrm{f}}+\mathrm{v}_{\mathrm{c}}$, soit $\mathrm{V}=\pi\left(\mathrm{d}^{2} \mathrm{e} / 4+\mathrm{dhe}\right)=\pi \mathrm{de}(\mathrm{d} / 4+\mathrm{h})$. Une casserole de dimension double $\left(\mathrm{d}^{\prime}=2 \mathrm{~d}\right.$ et $\left.\mathrm{h}^{\prime}=2 \mathrm{~h}\right)$ mais de même épaisseur e, est donc constituée d'un volume de cuivre égal à $\pi 2 d \mathrm{e}(2 d / 4+2 h)=\pi 4 \mathrm{de}(\mathrm{d} / 4+\mathrm{h})=4 \mathrm{~V}$ et elle est donc 4 fois plus lourde.

Elle déplace un volume d'eau 4 fois plus grand, mais la surface de son fond est elle-même 4 fois plus grande puisque de diamètre double. Ainsi la grande casserole s'enfonce exactement de la même hauteur que la petite bien qu'elle soit deux fois plus haute de bord: c'est celle qui apparaît comme flottant bien mieux. La grande casserole est à droite, sur les photos initiales de notre enquête. L'étude de la question demande ici le maniement minimal d'écritures algébriques et la connaissance qualitative de la loi d'Archimède. 
Qu'est-ce que cela permet de dire, pour les grands bateaux en fer? Sans doute, que plus ils sont grands mieux ils flottent et donc, plus ils peuvent porter de marchandises : ce que l'on constate en effet en allant observer un port de commerce et les porte-conteneurs. Mais bien sûr l'enquête devrait aussi être instruite par cette considération : on ne peut faire de grands bateaux pouvant porter beaucoup de conteneurs que parce qu'on sait les faire solides sans trop augmenter l'échantillonnage de leurs pièces c'est-à-dire en particulier, l'épaisseur de leurs parois, et parce que l'on sait fabriquer des moteurs suffisamment puissants sans être trop pesants. Enquêter sur cela demande des calculs que nous n'avons pas la place de développer ici, et quelques connaissances en résistance des matériaux. L'économie n'échappe pas aux effets d'échelle et les calculs qui font le lien entre le coût des infrastructures nécessaires au fonctionnement d'une tout et sa hauteur ne sont pas moins utiles à la détermination de sa bonne hauteur.

Les questions d'échelle sont si répandues en physique et biologie que nous ne saurions les épuiser, mais nous allons en citer d'autres, qui comme celles-ci pourraient devenir des "situations fondamentales pour ouvrir une enquête, et étudier les mathématiques qui modélisent ces phénomènes ».

Ainsi, on sait que le refroidissement d'un corps est proportionnel à sa surface d'interaction avec le milieu plus froid, ce qui permet de penser que, à forme et enveloppe isolante égale, plus un corps est petit plus il se refroidit aisément lorsque la température ambiante diminue. C'est sans doute pourquoi les petits animaux à sang chaud doivent avoir un métabolisme plus fort, afin de maintenir leur température interne constante, et pourquoi les serpents doivent être des animaux à sang froid qui ne s'animent que par les temps de bonne chaleur. Inversement, les éléphants ont du mal à réguler leur température et ils utilisent pour cela un dispositif inattendu, leurs oreilles fortement irriguées de sang.

Ces questions ne figurent jamais dans les curriculums de physique ou de biologie, parce qu'elles sont trop simples et qu'il est fastidieux de les étudier au cas par cas : elles relèvent d'un seul phénomène aisément décrit d'une phrase, les effets d'échelle. Mais en mathématiques elles peuvent donner lieu à de multiples études et enquêtes, dès la fin de l'enseignement élémentaire ou au tout début du Collège et jusqu'au Lycée selon la manière dont elles sont abordées. Ces enquêtes peuvent fonder la question de l'échelle comme généralisation du raisonnement sur les grandeurs proportionnelles aux cas où justement les phénomènes ne varient plus selon les mêmes lois parce qu'interviennent des dimensions multiples d'ordre 2, 3, etc. Ainsi en va-t-il dans l'étude des arbres et de leur respiration, qui peut s'outiller d'une enquête sur leur surface d'échanges avec l'atmosphère sachant leur encombrement, la densité de leurs feuilles au mètre cube, et la surface d'une feuille; ou dans l'étude des forêts et de leur intervention dans le recyclage du gaz carbonique. Ces enquêtes peuvent ouvrir sur la notion de dimension fractale d'un objet et être associées à l'étude de la respiration pulmonaire. Tout ce travail peut être conduit avant l'entrée des élèves dans le questionnement de l'analyse mathématique qui commence avec l'étude des fonctions de degré 2 et s'arrête trop souvent en ce point initial, car il donne de sérieux atouts pour comprendre ce que sont les fonctions et l'intérêt d'étudier leurs variations.

On peut de même anticiper sur les phénomènes dont un modèle est exponentiel parce que leur variation est proportionnelle à leur valeur actuelle. Ce serait un axe important de la formation des professeurs de mathématiques de tous niveaux que d'explorer tout cela et d'en tirer des occasions d'enseignement contextualisées parce que fondées sur une enquête préalable dans le voisinage matériel, social, culturel, technique, des élèves. 


\section{Conclusions, ouvertures théoriques et pratiques}

L'identification des questions porteuses des savoirs d'usage générique que l'on voudrait que les élèves apprennent à maîtriser est difficile, parce que ces savoirs ne vivent que dans des situations sociales qu'il faut transporter dans l'école comme situations didactiques fondamentales. Brousseau et Balacheff (1998) ont développé cette notion pour un enseignement des mathématiques orienté à l'époque par la réforme moderniste des années 1970-1990. Nous devons aujourd'hui reprendre son apport en imaginant des situations pour l'apprentissage de mathématiques contextuelles, qui sont celles qu'attendent les élèves d'aujourd'hui et leurs parents. Ce mouvement serait pour eux le signe fort que l'école cherche à les instruire pour eux-mêmes et non pas pour qu'ils deviennent les enfants sages et dociles de la République.

Sur ce chemin, nous rencontrons immédiatement de grandes et fortes questions qui se posent dans toutes les disciplines scientifiques, jusqu'à la sociologie et l'économie qui sont introduites au Lycée. Cependant, l'organisation de leur étude demande des connaissances anthropologiques assurées sur les pratiques sociales effectives dans les familles des élèves, depuis la question de la mesure et de l'évaluation du temps (qui va de la seconde aux saisons) aux questions plus générales du mesurage des grandeurs de différentes espèces et des techniques actuelles qui sont souvent opaques : comment compte-t-on les arbres d'une forêt, les participants à une manifestation, les poissons d'un lac ou d'une mer, les oiseaux migrateurs, comment mesure-t-on la hauteur du niveau de la mer, mais aussi l'âge d'un squelette d'hominidé, comment date-t-on une couche géologique, la naissance d'une étoile, comment évalue-t-on la distance d'une galaxie ? Mais d'abord, et cela peut être fait dès l'école élémentaire, comment mesure-t-on la hauteur d'une montagne, la distance d'une île que l'on peut voir au loin, la courbure de la terre et son rayon sachant la distance de l'horizon, le débit d'un pipe-line, le nombre des globules dans le sang d'un animal, etc. Rien que sur le comptage, nous nous apercevons que même les adultes instruits ne savent pas les procédés actuels, alors qu'ils devraient pouvoir les proposer à l'enquête des élèves en sachant ce qu'il y a à apprendre de telle ou telle technique.

Mais sur le mesurage des grandeurs continues les questions sont bien plus délicates et je n'en retiendrai qu'une qui peut servir de symbole à cette difficulté : "Comment mesurer (en pratique et en théorie) une ligne courbe, une surface bombée, ou un volume complexe comme celui d'un navire? » Nous ne parlerons pas du travail arithmétique qui fonde l'usage des nombres comme codes ou noms propres des objets industriels et qui assurent leur « traçabilité » dans une production mondialisée.

On s'aperçoit que tenter de répondre à de telles questions suppose d'entrer dans des pratiques fondées sur des mathématiques mais qui chaque fois sont solidement assises sur la connaissance d'un monde d'action et de pensée dont il faut connaître le langage non mathématique (Silvy, Delcroix et Mercier, 2013). Ainsi, les vols de migrateurs ne peuvent être observés que si l'on connaît les parcours et les dates des migrations, ce qui demande l'organisation de réseaux d'observation coordonnés, et déjà l'idée que les hirondelles africaines sont les mêmes que les hirondelles européennes demande la description précise des sujets de cette espèce, autant par leur vol ou leurs habitudes que par leur forme. Mais de même, l'observation des astres qui engage aux raisonnements astronomiques élémentaires : « Où est le Soleil lorsque l'on observe la pleine Lune ? » demandent des mois d'observation organisée avant toute tentative de modélisation géométrique du triangle Terre Lune Soleil, de 
ses angles et de ses déformations (Noirfalise et Matheron, 2009). Il faut à un tel observatoire une orientation de l'espace et le jeu de va-et-vient des repères absolu et terrestre, dont l'utilité et l'usage matériel et langagier n'apparaissent pas immédiatement. Nous imaginons donc une école bien différente du dispositif particulièrement bureaucratique actuel, qui gère les cohortes d'élèves en fonction de critères normalisés et qui les traite selon des rythmes quotidiens hebdomadaires et annuels particulièrement rigides.

Plus que la résolution de problèmes, dont discute à l'infini s'ils doivent être «concrets » ou " mathématiques " et même « ouverts », et qu'il faudrait résoudre pour ensuite étudier les techniques de leur résolution, nous proposons qu'une école démocratique soucieuse de la formation de citoyens instruits (capables de s'engager dans une enquête sur les problèmes qu'ils rencontreront et devront résoudre, mais que nous ne connaissons pas) leur propose d'enquêter sur des questions vastes et à rendre compte de leurs résultats afin d'engager un débat sur ce qu'il serait utile de savoir et d'apprendre pour aller plus loin vers une réponse utile.

Cette École là devra disposer de situations types qui auront été éprouvées, de manière à identifier les savoirs qui auront ainsi été enseignés et devront être appris. Elle saura en renouveler le stock au fur et à mesure de l'évolution de la vie sociale et de l'évolution technique. L'usage de ces situations devra pouvoir être connu, par une analyse épistémologique partagée, de l'enquête sur des pratiques à leur étude et à l'expérimentation conduite sous l'autorité des premiers modèles que l'on aura construits, puis aux moyens d'en rendre compte aux fins d'apprentissage, d'évaluation, d'amélioration, mais en tous cas pour que ces pratiques ne soient pas opaques et que leur transparence puisse être une revendication unanime. On sait que l'un des enjeux des travaux en didactique contextuelle conduits en Guadeloupe (Delcroix, Cariou, Ferrière et Jeannot-Fourcaud, 2015) est de relever ce défi, et on ne peut que s'en féliciter. Mais si la question semble aller de soi sur des questions d'écologie ou de géographie, elle est particulièrement difficile pour les sciences dont les objets semblent génériques comme les mathématiques ou la physique, et particulièrement sensible pour les questions de la langue. On peut cependant espérer qu'une organisation moins bureaucratique et plus démocratique de l'école puisse voir le jour, et penser que ce sera plutôt à la périphérie, là où le besoin est d'autant plus grand que la norme est plus faible.

\section{Références bibliographiques}

Bodin A. et de Franche-Comté I. (2006). Ce qui est vraiment évalué par PISA en mathématiques. Ce qui ne l'est pas. Un point de vue français. Bulletin de l'APMEP, 463, 240-265.

Bodin A. (1993). EVAPM. Fin de quatrième 1991. Fin de troisième 1992. Observatoire de l'enseignement des mathématiques. Une étude de l'APMEP. Paris : APMEP. Adresse : http://publimath.irem.univ-mrs.fr/biblio/AAP97018.htm [Consulté le : 6 septembre 2014].

Briand, J. et Peltier, M.-L. (2003). Étude de la disme de Stevin de Bruges. Adresse: https://halshs.archives-ouvertes.fr/halshs-00495125/ [Consulté le : 10 juillet 2015].

Brousseau, G. (1987). Fondements et méthodes de la didactique des mathématiques. Recherches en didactique des mathématiques, 7(3), 33-115.

Brousseau, G. (2002). Les grandeurs dans la scolarité obligatoire », Actes de la 11e Ecole d'Eté de Didactique des Mathématiques, Orléans, IREM D'Orléans, 31-348. Adresse : http://halshs.archives-ouvertes.fr/hal-00715071/ [Consulté le : 4 septembre 2014].

Brousseau, G. et Balacheff, N. (1998). Théorie des situations didactiques. Grenoble: Éditions la Pensée sauvage. 
Brousseau, G., Brousseau, N. (1976 [1987]) Rationnels et décimaux dans la scolarité obligatoire. Bordeaux: IREM de Bordeaux. Adresse : http://hal.archives-ouvertes.fr/hal00610769/ [Consulté le : 4 septembre 2014].

Brousseau, N. et Brousseau G. (1992). Le poids d'un récipient. Etude des problèmes de mesurage en CM. Grand N, n50, 65-87.

Carey, S. (2001). Cognitive foundations of arithmetic: Evolution and ontogenisis. Mind \& Language, 16(1), 37-55.

Chambris, C. (2010). Relations entre grandeurs, nombres et opérations dans les mathématiques de l'école primaire au 20e siècle : théories et écologie $»$, . Recherches en didactique des mathématiques, 30(2), 317-366.

Chevallard, Y. (1997). Familière et problématique, la figure du professeur. Recherches en didactique des mathématiques, 17, 17-54.

Chevallard, Y. (2007). «Les mathématiques à l'école: pour une révolution épistémologique et didactique »,. Bulletin de l'APMEP, 471, 439-461.

Chevallard, Y. et Bosch M. (2000). Les grandeurs en mathématiques au collège. Partie I. Une Atlantide oubliée. Petit x, 55, 5-32.

Dehaene, S. (2011). The number sense: How the mind creates mathematics. Oxford University Press.

Adresse :http://books.google.fr/books?hl=fr\&lr=\&id=SPaapa4PMVEC\&oi=fnd\&pg=P

R7\&dq $=++++++++++++$ Dehaene, + S. $+(1997) .+$ The+number+sense+.+New + York: + Ox ford+University+Press\&ots=YAxv2Uk6DX\&sig=r8ppkAXXZ3I11nwRuMMJN1lwwf 4 [Consulté le : 5 mai 2015].

Delcroix, A., Cariou, J.Y., Ferrière, H. et Jeannot-Fourcaud, B. (dir) (à paraître). Apprentissages, éducation, socialisation et contextualisation didactique: approches plurielles. Paris : L'Harmattan.

Dewey, J. (1958). Expérience and nature. New-York : Dover publications, DNC.

Deweys, J. (1916, [1975]) Démocratie et éducation: production à la philosophie. Paris: Armand Colin (traduction française ,1975).

Dutheil, C. (1996). Enfants d'ouvriers et mathématiques, les apprentissages à l'école primaire. Paris : L'Harmattan.

Fabre, M. (2015). Education et humanisme. Paris : Vrin.

Go, H. L. (2009). Des ingénieries didactiques de l'œuvre. Education \& didactique, 3(2), 7-45.

Izard, V. (2006). Interactions entre les représentations numériques verbales et non-verbales: étude théorique et expérimentale, Paris 6. Adresse : http://www.theses.fr/2006PA066048 [Consulté le : 13 octobre 2014].

Le Maréchal, J.-F. et Mercier, A. (2006). L'intervention et le devenir des connaissances antérieures des élèves dans la dynamique des apprentissages scolaires ». Ecole et sciences cognitives $\mathrm{n}^{\circ}$ AF14. Adresse : https://hal.archives-ouvertes.fr/hal-00375074/ [Consulté le : 22 novembre 2015].

Ligozat, F. (2008). Un point de vue de didactique comparée sur la classe de mathématiques: étude de l'action conjointe du professeur et des élèves à propos de l'enseignement/apprentissage de la mesure des grandeurs dans des classes françaises et suisses romandes. Mémoire doctoral des universités de Genève et Aix-Marseille.,

Mercier, A. (1994) Des études didactiques pourraient-elles aider l'enseignement des savoirs professionnels? Le cas des mathématiques dans les pratiques professionnelles. Disdascalia, 4, 5-20.

Mercier, A. (2014). Savoirs, compétences. Approche comparative de l'organisation des contenus et des formes de l'étude: variations et constantes disciplinaires, institutionnelles et culturelles. Dans F., Chnane-Davin et J-P. Cuq (dir). Approche comparative des savoirs et des compétences en didactique (p. 15-38). Paris : Éditions 
Riveneuve.

Noirfalise, A. et Matheron, Y. (2009). Enseigner les Mathématiques a l'école primaire. Géométrie, grandeurs et mesures. Paris : Vuibert.

Pugibet, C., Adam A. et Gason P. (1948). Arithmétique. Cours supérieur et classe de fin d'études, Certificat d'études primaires. Paris : Armand Colin.

Silvy, C., Delcroix A. et Mercier, A. (2013). Enquête sur la notion de «pedagogical content knowledge », interrogée à partir du "site local d'une question». Education \& didactique, 7(1), 33-58.

Stevin, S. (1585). L'arithmétique. Adresse : http://p olib.univ-lille3.fr/data/015index.html

Toullec-Théry, M. et Marlot, C. (2013). Les déterminations du phénomène de différenciation didactique passive dans les pratiques d'aide ordinaire à l'école élémentaire ». Revue française de pédagogie, 182(1), 41-54. 